\title{
LA INTEGRACIÓN WI-FI/CELULAR, EVOLUCIÓN Y DESAFÍOS
}

Chiozza, Juan A.; Valdez, Alberto D.; Miranda, Carlos A.; Schlesinger, Paola L.; Miranda, Carlos V. (*)

\section{RESUMEN}

La demanda de datos móviles ha disparado en los últimos años muchos avances en las comunicaciones inalámbricas. La red celular con sus sucesivas generaciones tiende indefectiblemente a un servicio de datos, las redes $\mathrm{Wi}-\mathrm{Fi}$ enfocadas en los dispositivos denominados portátiles es exclusivamente de datos. Pero ninguna por si sola puede asegurar una cobertura inalámbrica de calidad en todos los ámbitos. La interacción de ambas en los dispositivos a mejorado la experiencia del usuario pero lejos está de ser óptima. La integración de estas redes con protocolos y sistemas enfocados en sacar lo mejor de ambas todo el tiempo es lo que se trata en este trabajo. El estado actual del arte y las proyecciones futuras se abordan dando una idea de presente y futuro de la integración.

Palabras claves: WiFi, celular, integración, Hotspot 2.0, ANDSF.

\section{INTRODUCCIÓN}

El objetivo del presente trabajo es presentar la evolución de la integración entre las redes celulares y Wi-Fi (marca comercial que representa las tecnologías 802.11x) para mejorar la experiencia del usuario al utilizar el servicio de datos.

La creciente demanda de aplicaciones y servicios que dependen de éstos en los móviles, ha llevado a saturar las redes celulares, sobre todo en áreas de alta densidad de usuarios. Muchos operadores han recurrido a la integración del servicio de datos alternativo a través de las redes $\mathrm{Wi}-\mathrm{Fi}$, sean estas abiertas o integradas al mismo operador. El desafío ante el vertiginoso crecimiento de la demanda es poder hacer un uso inteligente de ambas redes manteniendo la transparencia para el cliente y mejorando la QoE (Quality of Experience: Calidad de la Experiencia).

El informe se ha estructurado de la siguiente manera: En la sección 1 se da una idea del tema que se tratara en el resto del trabajo.

La sección 2 se divide en varias sub-secciones para poder organizar y abordar los temas necesarios de manera estructurada y relacionada según su importancia. Así, en la sección 2.1 se da un panorama de como ha sido la evolución del tráfico inalámbrica responsable de incentivar y acelerar la integración. La sección 2.2 relata y resume lo

$\left({ }^{*}\right)$ Facultad de Ciencias Exactas y Naturales y Agrimensura, Departamento de Ingeniería, UNNE, Corrientes CP3400, Argentina.dvaldez@exa.unne.edu.ar 
acontecido en cuanto a la integración WiFi/Celular en sí y su evolución, surgen de ese desarrollo los desafíos que se deben sortear, los que se detallan en la sección 2.3. Como se mencionara, la integración conlleva a la necesidad de administrar y coordinar redes heterogéneas, tema abordado en la sección 2.4. Esta sección contiene además una sub-sección dedicada al estándar que implementa la integración desde la red celular. En la sección 2.5 se explican los mecanismos de la integración desde el punto de vista del equipo móvil y las herramientas que este debe implementar. La red Wi-Fi y sus herramientas dedicadas a la integración y mejor movilidad de sus usuarios son explicadas en la sección 2.6, para finalizar el desarrollo con la sección 2.7 donde se analiza la arquitectura combinada de $\mathrm{WiFi}$ /Celular. Después de la introducción y el desarrollo, finaliza el trabajo con la sección 3 donde se expone nuestro punto de vista del estado actual del arte y de la proyección a futuro que tiene la integración de las redes $\mathrm{Wi}-\mathrm{Fi} /$ Celular.

\section{DESARROLLO}

\subsection{Evolución del tráfico inalámbrico} [1][2]

La evolución del tráfico cursado en forma inalámbrica ha crecido más allá de todas las previsiones debido a distintos factores. La evolución de los dispositivos móviles (teléfonos inteligentes, tabletas, etc.) ha disparado la aparición de aplicaciones que utilizan conexión de datos, los streaming (torrentes) de video y audio, la globalización de las redes sociales, videoconferencias, y la continuidad del uso de estos dispositivos en el hogar y también en lugares públicos, y empresas.
Sumado a estos factores, el crecimiento de los servicios en la nube implica en muchos casos un uso extendido del dispositivo no sólo como medio de comunicación u ocio sino además como herramienta de trabajo.

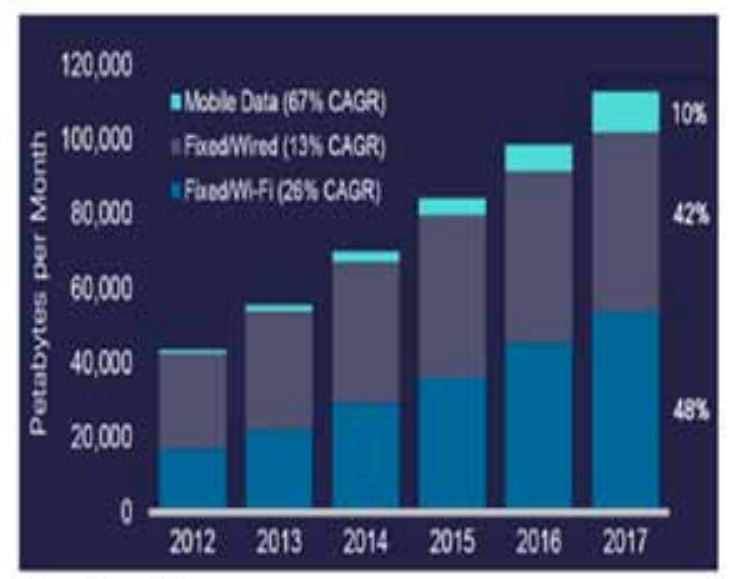

Source: Cisco WNI

Figura 1. Tráfico IP global ${ }^{2}$

En definitiva, la red de datos inalámbricos debe cubrir cada lugar y a la vez tener la capacidad y eficiencia necesaria para poder brindar el servicio requerido a un costo razonable. Partiendo del 2011, en el corriente 2015, se espera que el tráfico de datos inalámbricos se multiplique por un factor de $25^{1}$ a nivel mundial.

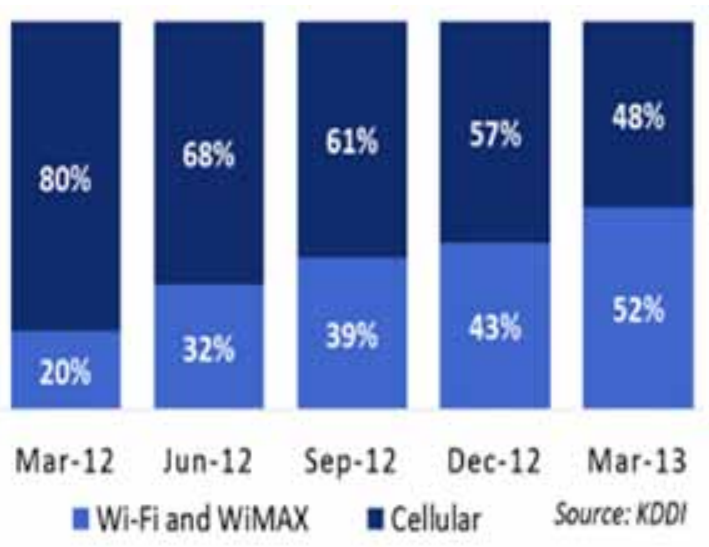

Figura 2. Tráfico Wi-Fi y WiMAX como porcentaje del tráfico en celulares - KDDI 2 
La Figura 1 muestra con más precisión la evolución que ha tenido el tráfico inalámbrico y más importante en particular el que se cursa a través de redes $\mathrm{Wi}-\mathrm{Fi}$. Si bien por la eficiencia de $4 \mathrm{G}$ (cuarta generación de la figura 1 . Tráfico IP global ${ }^{2}$ red celular) en cuanto al tráfico IP (Internet Protocol: Protocolo de Inter-red), se espera un incremento del porcentaje para la red celular, Wi-Fi seguirá siendo claramente quien lleve la mayor porción del tráfico inalámbrico. Cabe aclarar que el tráfico Wi-Fi a que refiere la figura 1. resulta de considerar conjuntamente los tráficos de todas las variantes de tráfico $\mathrm{Wi}-\mathrm{Fi}$, es decir público tarifado, público abierto, empresas, redes hogareñas, etc. Por otra parte, en la Figura 2., está representado el tráfico sobre Wi-Fi y WiMAX (Worldwide Interoperability for Microwave Access: interoperabilidad mundial para acceso por microondas) en porcentaje del tráfico en celulares sobre la red del operador japonés KDDI Corporation (Corporación KDDI). Puede verse el crecimiento constante del tráfico por las redes alternativas a la propia red celular. Esto remarca lo dicho en cuanto a la evolución de la demanda de la red alternativa a la celular.

\subsection{Evolución de la integración $\mathrm{Wi}-\mathrm{Fi} /$ Celular [1][2]}

El cubrimiento de la red celular para el tráfico de datos puede ofrecer un buen servicio en ciertas zonas, sobre todo las más cercanas a los sitios de emplazamiento de las antenas y dependiendo de la densidad de usuarios que demanden este servicio. En áreas cubiertas, o de alta densidad de equipos móviles activos el nivel de tráfico ofrecido decae notablemente. Por ello ha cobrado cada vez más importancia que estos equipos puedan conectarse alternativamente al ser- vicio de datos vía canal celular, o vía $\mathrm{Wi}$-Fi. Las redes Wi-Fi utilizan el mismo estándar en todo el mundo y además ocupan bandas no licenciadas del espectro. Estas características, sumada a la inclusión de interfaces de redes $\mathrm{Wi}-\mathrm{Fi}$ en todos los equipos portátiles (netbook, notebook) y móviles (celulares 3G y posteriores, tabletas), ha impulsado la proliferación de redes $\mathrm{Wi}-\mathrm{Fi}$ en todo el mundo y en los ámbitos más diversos, desde la red hogareña paga hasta la red abierta en lugares públicos cerrados o al aire libre.

Algunos prestadores de servicio celular incluso han implementado redes $\mathrm{Wi}-\mathrm{Fi}$ propias accesibles para sus usuarios de manera de asegurar la cobertura de datos en lugares donde la red celular no puede brindarlos apropiadamente.

Hay que tener presente que la experiencia para el usuario debería ser tal qué no distinga a que red está conectado ni tenga que hacerlo manualmente, como así también las aplicaciones no tendrían que sufrir retardos, interrupciones o bloqueos al cambiar de un sistema a otro. Es decir debería ser totalmente transparente al usuario y a las aplicaciones que éste ejecuta en su equipo. Por ejemplo podría reproducir sin interrupciones un streaming de video al alternar entre tales redes.

Este requisito conlleva una serie de cuestiones que se deben tener en cuenta para poder lograrlo y es lo que ha sido material de desarrollo continuo en los últimos años para hacer cada vez más satisfactoria la integración $\mathrm{Wi}-\mathrm{Fi} /$ Celular, tanto desde el punto de vista del cliente como en los costos de instalación y operación del prestador.

En la actualidad el esfuerzo conjunto de 
1a IEEE (Institute of Electrical and Electronics Engineers: Instituto de los Ingenieros Eléctricos y Electrónicos), WFA ( $W i-F i$ Alliance: Alianza Wi-Fi), y WBA (Wireless Broadband Alliance: Alianza banda ancha inalámbrica), han logrado estandarizar las características para que el Wi-Fi alcance el grado de transporte de datos (carrier), en lugar de actuar solamente en el último tramo hacia el cliente. Ejemplo de estos esfuerzos son Hotspot 2.0, un grupo de trabajo de la WFA cuyos programás se denominan Passpoint y NGH (Next Generation Hotspot: Punto de Acceso de Nueva Generación). Una de las principales ventajas de Hotspot 2.0 es la posibilidad de conectarse a una red utilizando credenciales del usuario que se encuentran en el equipo, por ejemplo el tarjeta SIM (Suscriber Identity Module: Modulo de Identidad del Abonado)

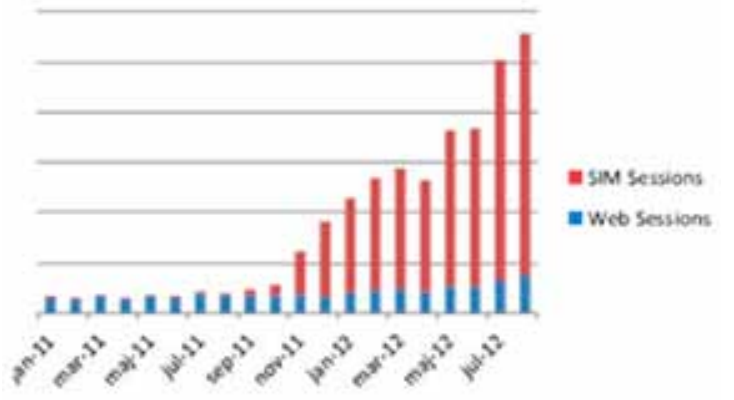

Figura 3. Tráfico Wi-Fi en aeropuertos luego de la introducción de la autenticación con SIM ${ }^{2}$

Esto permite que el usuario no tenga que introducir una clave para poder conectarse a un AP (Acces Point: Punto de Acceso) del mismo proveedor de servicios celulares, logrando un paso importante en el automatismo de esta tarea que mejora la transparencia para el usuario. Como ejemplo de las ventajas que esta solución ofrece véase la Figura 3. que representa la evolución del tráfico Wi-Fi en aeropuertos, discriminado según el tipo de autenticación utilizada por el equipo móvil. Se ve claramente como aumento el tráfico a partir del introducción de la autenticación basada en SIM que se hace sin intervención del usuario.

También se proveen nuevos mecanismos para que pueda accederse a información sobre los AP sin necesidad de asociarse previamente.

Otra ayuda en la evolución hacia una integración mayor es que en poco tiempo más no habrá distintos estándares operando las redes celulares, ya que claramente el estándar 4G es LTE (Long Term Evolution: Evolución a Largo Plazo) en todo el mundo, contrariamente a lo que pasaba con las generaciones anteriores donde en $2 \mathrm{G}$ y $3 \mathrm{G}$ coexistían estándares provenientes de distintas organizaciones.

Estaunicidad de 4G favorece no sólolainvestigación sino la implementación de redes y el diseño y fabricación de los móviles que responderán a un solo estándar mundial.

Hay también esfuerzos dirigidos a integrar las redes $\mathrm{Wi}-\mathrm{Fi}$ en el núcleo (core) de la red celular. Hoy por hoy, la red celular tiene su núcleo y la red Wi-Fi se conecta al servicio de datos fijo o a algún otro punto de acceso del proveedor. El hecho de lograr este objetivo redundaría en una reducción de costos de implementación y de operación, además de facilitar la facturación y el control de los clientes. La organización 3GPP (Third Generation Partnership Project: Asociación para el Proyecto de Tercera Generación), ha trabajado e invertido mucho esfuerzo en los últimos años para la evolución de la integración $\mathrm{Wi}-\mathrm{Fi} /$ celular. Más precisamente se han logrado un número de iniciativas tendientes a mejorar la selección desde los dispositivos móviles de la óptima red $\mathrm{Wi}-\mathrm{Fi}$, la integración de estos 
puntos de acceso al núcleo de la red celular (core), y la autenticación del usuario de celular mediante el acceso $\mathrm{Wi}$-Fi utilizando la información contenida en la SIM. $\mathrm{Mu}^{-}$ chas de estas iniciativas están incluidas en la estandarización de ANDSF (Acceses Network Discovery and Selection Function: Descubrimiento del Punto de Acceso y Función de Selección), que provee una estructura dentro del mismo core de la red celular, personalizable para el proveedor, de manera de dar información a los móviles para que puedan decidir el mejor punto de acceso donde conectarse, sea este de la red celular o o la red Wi-Fi. La publicación del documento 12 (Release 12) de la 3GPP [4], incluye justamente los esfuerzos por coordinar y alinear el funcionamiento de ANDSF desde el núcleo y el Hotspot 2.0 desde los móviles [6]. También, en este documento, se vuelca la evolución para lograr la preservación de la dirección IP del móvil aunque este conmute a distintos puntos de acceso. En definitiva los esfuerzos de 3GPP están encaminados a proveer las herramientas necesarias para que, implementadas por los operadores, el usuario del equipo móvil tenga una mejor experiencia al utilizar el servicio de datos en su dispositivo, independientemente y en forma transparente a las conmutaciones que se vayan dando entre el acceso de radio del cliente y la óptima red disponible. Con el acompañamiento a todos estos esfuerzos desde los constructores de equipos móviles, se logra combinar las bondades de la información de políticas que llegan desde la red del operador, para que el móvil de acuerdo a su software y esta valiosa información suministrada, decida la mejor conexión en tiempo real, mejorando notablemente la QoE del cliente.

\subsection{Desafíos de la integración $\mathrm{Wi}-\mathrm{Fi} /$} celular[2][3]

Para los lugares y usuarios con equipos móviles o portátiles que utilizan el servicio de datos en una forma semi-nómada, $\mathrm{Wi}$-Fi aparece como la opción principal, siendo su acceso rápido y simple. Pero cuando se trata de usuarios con más movilidad o que utilizan indistintamente la red celular o $\mathrm{Wi}-\mathrm{Fi}$, existen todavía problemás que complican la óptima selección en tiempo real de la red más conveniente. Se puede decir que estos problemas fundamentalmente son cuatro:

a- El problema de la selección prematura de Wi-Fi desde el móvil, cuando aun la conexión a la red celular es más ventajosa en velocidad de transmisión. El motivo es que los parámetros de selección son muy pocos y no tienen en cuenta precisamente la mejor opción en cuanto a velocidad y retardo, sino solo la opción preferir conexión $\mathrm{Wi}$-Fi.

b- Al seleccionar la red Wi-Fi puede que la ubicación la favorezca, pero el móvil desconoce que el tráfico que se cursa por el núcleo de esa red está saturado, provocando nuevamente una selección errónea de la mejor red al utilizar como parámetro de decisión el nivel de señal recibida (RSL: Received Signal Level).

c- Otro error al seleccionar o preferir la red Wi-Fi por su nivel de señal es que si bien esta red no está saturada su conexión al núcleo de red esta muy por debajo en cuanto a velocidad de transmisión de la que tiene el nodo celular. Así, nuevamente se cae en una degradación de la QoE del usuario móvil.d- Nuevamente sin tener en cuenta los desafíos anteriores, se puede pro- 
ducir un cambio intermitente de conexión en un escenario de condiciones de propagación con elevado desvanecimiento selectivo. Lógicamente, los retardos en el tráfico por el continuo cambio de conexión producen de esta forma una degradación de la QoE.

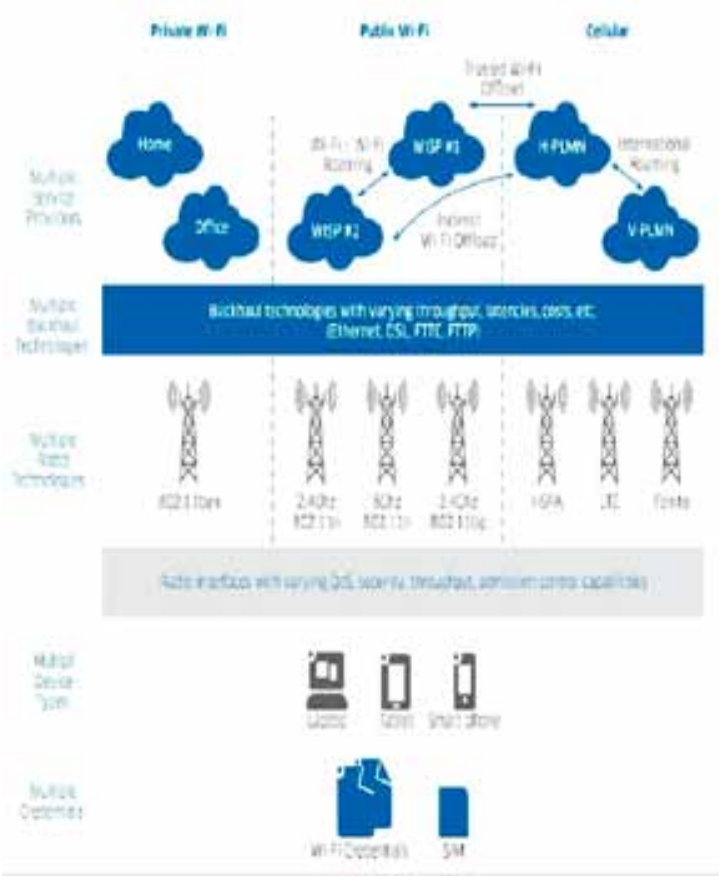

Figura 4. Roaming con ANDSF y Hotspot $2.0^{4}$

Como puede verse en estos cuatro casos, la integración eficiente de las redes Wi-Fi y celular tienen muchos aspectos a considerar y se tratan de solucionar mediante distintas herramientas disponibles para cada uno de los actores de esta integración. Hay una tarea que es fundamental al momento de la selección óptima pensando en una satisfactoria QoE, y es la recolección de información del estado de los distintos puntos de la red y sus características para poder entregar en tiempo real esta información depurada a cada equipo móvil, información con la cual el móvil podrá realizar una conexión óptima en cuanto al tráfico de datos.

\subsection{Coordinación de redes heterogé- neas [3][4]}

Es evidente que la coexistencia de los servicios prestados por la red celular y la red Wi-Fi a un equipo móvil o portátil se ha instalado definitivamente y necesitan convivir porque por sí solas no pueden prestar un servicio con alta QoE para el usuario actual que demanda servicio de datos continuo esté en el lugar que éste y lo haga en tránsito o en forma estática.

Por ello, por todo lo que se describió en los puntos anteriores, es inevitable pensar en redes heterogéneas (HetNet: Heterogeneus Network: Redes Heterogéneas). Un mismo UE (User Equipmment: Equipo de Usuario), tiene la posibilidad de conectarse a distinto tipos de redes para poder dar servicio a las aplicaciones que activa el propietario. Para coordinar las posibilidades desde la red celular, como se anticipó, existe la herramienta marco ANDSF, y desde las redes Wi-Fi el Hotspot 2.0. Se analizará en adelante la necesidad de políticas que coordinen los esfuerzos de estas dos herramientas para que la $\mathrm{QoE}$ del usuario sea cada vez mejor. Es fundamental entender el escenario de futuro inmediato con el que se debe trabajar, la Figura 4 muestra la clara complejidad del mismo.

Cada una de estas tecnologías y cada uno de los operadores que las controlan tienen sus políticas para por ejemplo seleccionar el punto de conexión más adecuado, priorización de tráfico, etc. Para el usuario que va moviéndose y pasando del entorno de influencia de una red a otra, el UE debe decidir a cuál conectarse, y para ello cuenta con información de cada una de estas redes que no precisamente tienen las mismas políticas para tratar los parámetros involucrados 
y tampoco consideran exactamente la misma cantidad y tipo de información al UE.

Esto es, no hay precisamente una consistencia entre estas políticas y eso degrada la QoE del usuario que podría ser mucho mejor de existir una coordinación y consistencia entre las políticas de las distintas redes y operadores.

Este escenario queda representado en la Figura 5, donde se incluyen las fuentes de información que deberá evaluar el gestor de conexión, CM(Connection Manager) del UE para encontrar la conexión óptima.

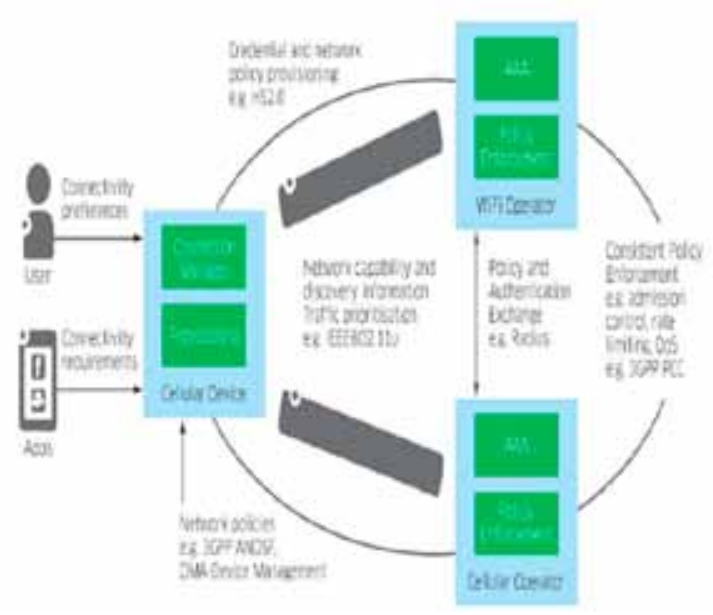

Figura 5. El rol del gestor de conexión del $\mathrm{UE}^{4}$

La tarea llegará al mejor resultado para los parámetros que dispone el $\mathrm{CM}$, pero en la realidad no será realmente la óptima de todas las disponibles. Ello se debe al complicado escenario y a las inconsistencias de las políticas que entran en juego llevando al CM a tomar una decisión no óptima.

Algunos operadores recurren a aplicaciones de terceros que incluyen en el UE para gestionar la conexión. Pero muchas veces la diferencia de escenarios que ve cada uno de ellos provoca un conflicto entre tales decisiones llevando nuevamente a una no óptima conexión.

\subsubsection{El sistema ANDSF de 3GPP [3] [4][6]}

Se analizarán en este apartado las características claves para entender el funcionamiento de ANDSF. Éste responde a una arquitectura cliente-servidor, localizándose el servidor en la red del operador celular, distribuyendo desde allí hacia los UE las políticas de intercalación a través de una interface denominada S14, basada en las especificaciones OMA-DM (Open Mobile Alliance - Device Management: Alianza móvil abierta - Gestion de dispositivos), o alternativamente con intercambio de XML (eXtensible Markup Language: Lenguaje de Marcas Extensibles) sobre TCP (Transport Control Protocol: Portocolo de Control de Transporte). La información se agrupa en el MO5 (Management Object: Objeto Gestionado), donde cada rama del objeto contiene la información especificada en forma de parámetro. La parametrización está implementada de manera qué, al enviarla al UE, su CM pueda decidir a que conexión y en que instante unirse para obtener una óptima QoE para el usuario. E1 UE está recibiendo en tiempo real continuamente información del servidor ANDSF, aunque esté en tránsito, de manera de ir cambiando de conexión a medida que se desplaza. ANDSF conoce la configuración de la red y su estado actual, información que vuelca en los parámetros del MO, que será enviada a cada móvil ajustado para la ubicación que este posee. Cada rama o nodo del MO tiene a su vez muchas variantes que son las que resume en el respectivo parámetro que caracteriza ese nodo para una ubicación de UE determinada. En 
tiempo real, los párametros que identifican las caracteristicas claves de la red, para obtener un punto óptimo de conexión, son actualizados permanentemente en el servidor ANDSF, que ademas conoce la topografía de la red en la que actúa. Con ésta información, la procesa y ajusta para cada móvil que pide conexión a la red o está en movimiento de manera de entregarle los páramteros para sus condiciones en particular.

\subsubsection{Estructura de alto nivel del MO}

En la Figura 6 se ve la estructura de alto nivel del MO con sus nodos principales. Para un detalle de la información y ramificación de cada nodo puede verse la norma que lo define en [5], no obstante a continuación se describe la información que resume cada nodo principal del MO.El nodo que contiene las políticas de movilidad entre sistemas es el ISMP (Inter-System Mobility Policies: Políticas de Movilidad Internas del Sistema) que enrutará el tráfico IP sobre un único enlace de radio, cuando el móvil no tenga capacidad de llegar al núcleo de la red por más de un acceso a la vez. Algunas de las reglas son definir qué tipo de acceso es preferible sobre otro en el área de influencia del UE, sea de una tecnología sobre otra o dentro de una misma tecnología los distintos accesos dentro del rango del UE. También se pueden asignar bandas horarias, o en fechas determinadas, en las qué, un acceso es preferible sobre otro de acuerdo a las estadísticas de tráfico que posee el operador.

El nodo de ANDI (ANDI: Acces network Discovery Information: Información para el descubrimiento del acceso), es utilizado para enviar información al UE de los acceso de redes que están disponibles para los UE que están en la cercanía. Además de incluir información sobre la tecnología de cada nodo, también se envía la correspondiente etiqueta de identificación. Para poder evaluar estos parámetros ANDSF se basa principalmente en la geolocalización de los UE.

Para gestionar la ubicación de los UE se utiliza el nodo UE_Location (UE_Location: Localizacion del UE). La actualiza-

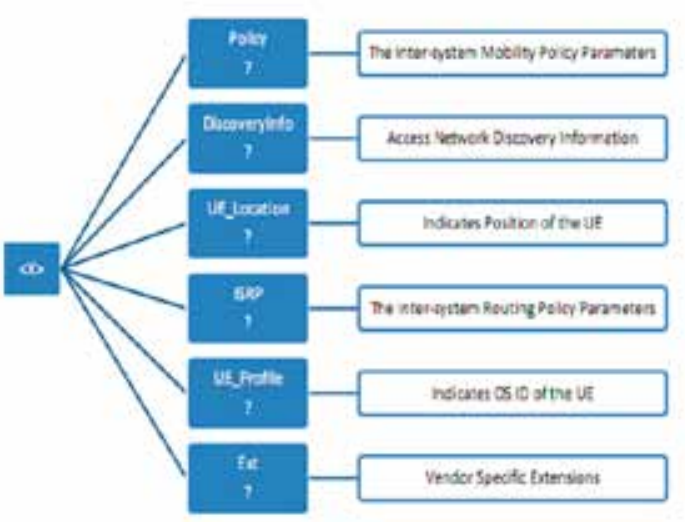

Figura 6. ANDSF MO - Estructura de alto nivel ${ }^{3}$

ción de la información puede ser activada por la pérdida de validez de la información o por un cambio en la posición del UE.

El nodo ISRP (Inter-system Routing Parameters: Parámetros de Ruteo Entre sistemas), es el que marca las políticas de ruteo cuando el UE tiene la capacidad de enviar tráfico IP por más de un acceso de radio simultáneamente. Éste nodo maneja mucha más información y tiene distintas variantes de orientación del tráfico del UE, las que conforman cada una un subnodo del ISRP. Las variantes son ForFlowBased (basada en el flujo), ForServiceBased (basada en el servicio) y ForNonSeamlessOffload (orientado a la derivación de tráfico sin interrupciones)[5]. 
El ISRP puede enviar una o más de estas variantes al UE. Dependiente de la aplicación corriendo sobre IP esta demandara alguno de los servicios nombrados cuyas reglas para accederlos son provistas al UE por el ISRP. En oportunidades puede existir más de una posibilidad dentro de cada servicio o subnodo del ISRP, cada uno de los cuales tiene a su vez opciones de $\mathrm{co}^{-}$ nexión de distintas tecnologías cuya validez dependerá del criterio de ruteo (routing criteria) y de las reglas de ruteo (routing rules) contenidas en cada servicio nombrado.

Es importante tener en claro que quien decide la conexión es el CM del UE, a quien el sistema cliente-servidor ANDSF le apor- ta su amplio conocimiento de las condiciones de la red. Para tener idea de la complejidad del armado de la información enviada al UE, en la Figura 7 se muestra parte de un envío del ISRP al UE desde el ANDSF local (H-ANDSF:home ANDSF: ANDSF Local) el detalle del subnodo ForFlowBased para indicar al UE la información de conexión requerida para las aplicaciones que éste solicitó, en este ejemplo Skype, Youtube y Facebook, abreviadas en la Figura 7 como SKP, YTB.com y FBK respectivamente.

Allí se pueden ver las distintas opciones de conexión, los criterios y reglas de ruteo para cada posibilidad, prioridades, etc.

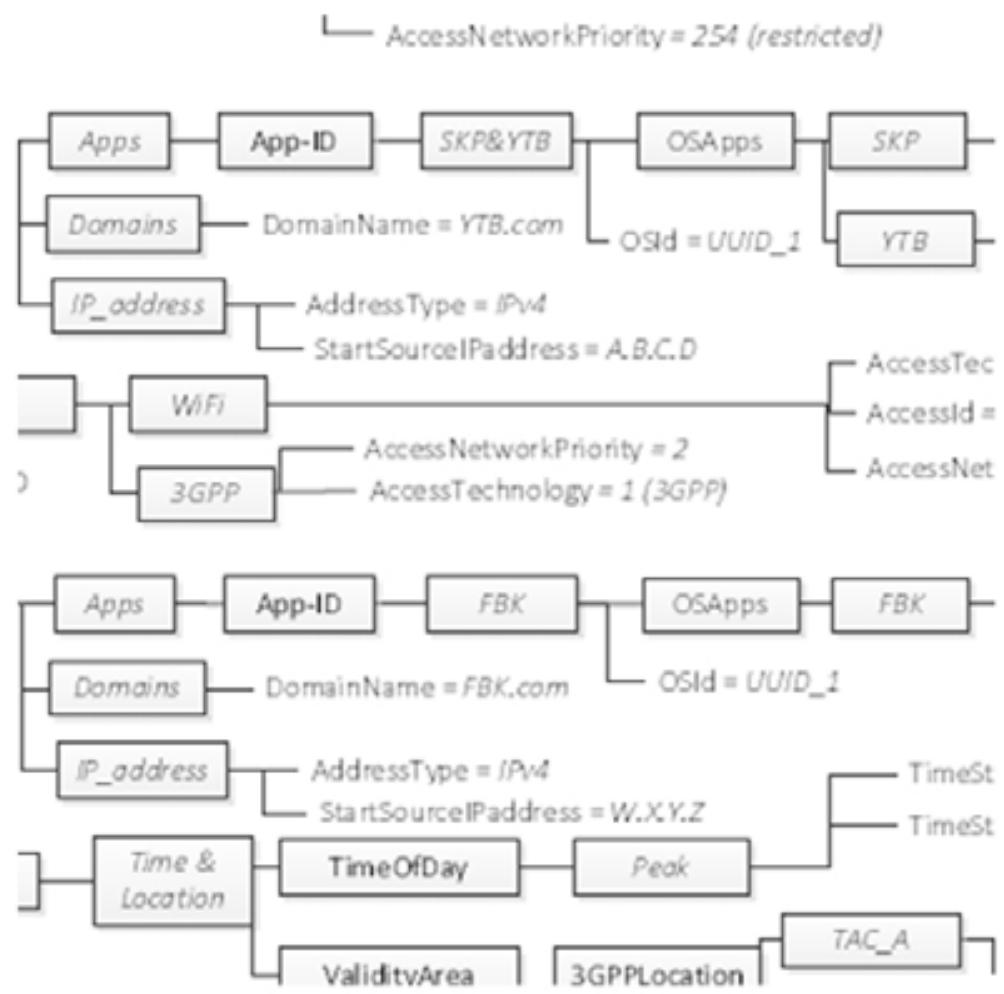

Figura 7. Parte de la configuración del subnodo ForFlowBased por el H-ANDSF 
Los escenarios del UE definen que regla deberá aplicar.

Dentro del esquema enviado, las primeras dos bifurcaciones del diagrama están etiquetadas como Home (local) y Roaming (en tránsito). La primer regla se aplica si el UE está dentro del área de cubrimiento del H-PLMN (Home-Public Land Mobile Network: Red Móvil Publica Terrestre-Local). Si el UE en tránsito cambia a un área en la que es visitante y la red no tiene servidor ANDSF, se aplican las reglas englobadas en la bifurcación Roaming. El último escenario es cuando se encuentra como visitante en una red y ésta si tiene servidor ANDSF, en cuyo caso el UE establecerá comunicación con el servidor y recibirá las reglas a utilizar para conexión de datos dentro de su red. El ISRP es claramente el nodo más complejo del MO.

En la Figura 7, cada rama principal está etiquetada con la sigla IFOM (IP Flow Mobility: Movilidad del Flujo IP), seguida de un número que indica las distintas posibilidades de conexión. Cada una de estas posibles conexiones tiene una Rule Priority (Regla de Prioridad) siendo prioritario el de menor número. Es decir que al tener el UE demanda de alguna aplicación dará prioridad de atención según este número. También tienen la bifurcación RutingCriteria (Criterio de Ruteo), donde en este caso tiene la etiqueta TimeÉLocation (tiempo y Localización), formada a su vez, por dos etiquetas, TimeofDay (Hora del día) y ValidityArea (Área de validación). En la primera se establece el horario del día dentro del que se produce el pico de utilización de esa conexión.

Con esta política establecida de acuerdo a los registros estadísticos que tiene el ANDSF, el operador evita la congestión de tráfico indicando a los UE que preerentemente no utilicen esa conexión en un horario determinado. La segunda etiqueta da el área de validez de la política. Por ejemplo puede indicar que la regla es válida si esta en dentro del área de PLMN =10010, lo que significa : dirección de la PLMN = 10010. Si está en otra área esta regla no es válida.

Siguiendo con la descripción de los demás nodos principales, está el nodo UE_Location (localización del UE), que contiene información acerca de la ubicación geográfica y dentro de la red del dispositivo.

La información de configuración del sistema operativo del UE esta contenida en el nodo UE_Profile (perfil del UE), esta información es enviada al servidor ANDSF al establecer la conexión. Cualquier información o nodo adicional proporcionado por el UE que no es soportado por el servidor es ignorado por éste. Finalmente, dentro del más alto nivel de la estructura del MO, está el nodo Ext_node (nodo externo), que contiene información del ANDSF MO específica del fabricante del equipo y no es alcanzada por la norma de 3GPP.

\subsection{El dispositivo móvil UE actual}

Actualmente, los dispositivos UE, se conectan a una WLAN (Wireless LAN: LAN inalámbrica), de distintas formas utilizando soluciones propietarias, sin conectarse al núcleo de la red. Por ejemplo algunos UE se conectan a una WLAN ni bien están dentro de su alcance y en el caso de tener más de una señal apta, recurren a alguna aplicación propietaria para resolver la conexión. Normalmente utilizan algún algoritmo para decidir, en base a información recolectada por el UE, es decir son aplicaciones basadas en el cliente. Algunos de esos parámetros son 
los valores de RSL (Received Signal Level: Nivel de Señal Recibida), estadísticas de acuerdo a la MAC del AP, etc. También en algunos casos, la elección se deja al usuario en forma manual, y en otras alguna aplicación de capa superior decide la conexión. Esto último puede entrar en conflicto con los métodos de selección de capas inferiores que normalmente lo hacen en base a la mejor señal recibida. Muchas de este tipo de aplicaciones para automatizar el proceso de elección del AP al cual conectarse tienen inconsistencias para seleccionar la mejor opción, ya que no manejan parámetros del AP hacia adentro de la red, como capacidad de tráfico, estado de carga de su interconexión con el núcleo, etc.

En la Figura 8 se ve el esquema general de funcionamiento de un UE de uso común en la actualidad y sus funcionalidades de conexión. Como se ve en general los UE para poder decidir la conexión a realizar lo hacen

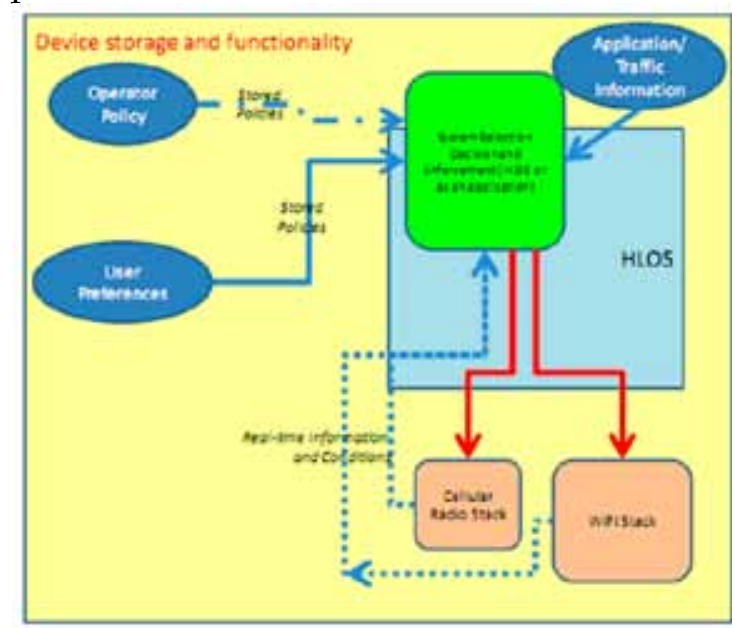

Figura 8. Funcionalidades de los UE actuales ${ }^{3}$

mediante alguna aplicación específica o algoritmo incluido en el sistema operativo del dispositivo (HLOS:High Level Operating System: Sistema Operativo de Alto Nivel). Para ello pueden utilizar políticas asignadas por el operador al configurar el UE (Operator Policy), configuración del usuario (User Preferences, y algunos parámetros que el UE puede recolectar y utilizar para hacer estadísticas de conexiones anteriores, señal recibida, y con ellos decidirse por uno de los puntos de acceso Wi-Fi o celular.

Con la utilización del servidor ANDSF, los UE podrán recibir la información parametrizada en el MO y enviada con el formato de la interface S14, que se agregaría en el esquema de la Figura 8, aportando sus datos a la parte identificada como Operator Policy. Así la interface S14 interactúa con las políticas del operador configuradas al habilitar el terminal UE, y con ello influyen directamente en la decisión del CM para encaminar el tráfico IP.

Como se viera en la descripción del MO dependerá del tipo de UE si puede utilizar las dos opciones de radio, es decir cursar tráfico simultáneamente por las interfaces de radio de Wi-Fi y de Celular aprovechara la información contenida en el nodo ISRP. Si por el contrario su tecnología sólo le permite utilizar una interface de radio por vez, aprovechará las indicaciones del ANDSF contenidas en el ISMP.

\subsection{El estándar IEEE 802.11u [3][6]}

Conocido como Hotspot 2.0 (HS2.0) o Wi-Fi Certified Passpoint (Wi-Fi Punto de Paso Certificado), ésta norma define los mecanismos necesarios para que un $\mathrm{UE} o$ cualquier dispositivo con conexión $\mathrm{Wi}-\mathrm{Fi}$, pueda al entrar al área de un proveedor tener un roaming (movilidad entre puntos de acceso sin perder conexión) similar al de la red celular. Esto es una gran mejora respec- 
to de la movilidad incipiente que tenían las redes $\mathrm{Wi}-\mathrm{Fi}$ con un proceso de roaming que sólo podía asegurar conectividad pero no como una sesión sin cortes (seamless session) como puede ofrecer con HS2.0.

Las redes Wi-Fi con HS2.0, distribuyen la información parametrizada en forma también de un MO como se vio en ANDSF, estos datos son utilizados por el UE o cualquier equipo con movilidad, para decidir su conexión óptima en cuanto al tráfico IP.

De esta manera los equipos celulares UE que soporten tanto ANDSF como HS2.0 tendrán una mejor QoE para el usuario IP. También da la posibilidad a los operadores de redes celulares de integrar en sus redes nodos AP conectados al propio núcleo de red, y como HS2.0 permite la autenticación automática por SIM, esto no solo mejora la QoE del usuario, sino que permite al operador tarifar este servicio que de otra forma se perdería en otro operador o en una red abierta de menores prestaciones pero con cobertura de radio en zonas críticas.

$\mathrm{Al}$ encontrar una señal de $\mathrm{Wi}-\mathrm{Fi}$, se activa el GAS (Generic Advertismet Service: Servicio Genérico de Anuncio), que es un servicio de capa 2 , para que aplicaciones de capas superiores puedan intercambiar información con el $\mathrm{AP}$ o un servidor antes de la autenticación y registración. Este permite que entre en acción el ANQP (Acces Network Query Protocol: Protocolo Consulta de Acceso a la Red), protocolo que se encarga de consultar al servidor de la red WiFi la información necesaria para poder seleccionar la mejor red o punto de acceso.

Como se comentó en párrafos anteriores, ésta es la parte similar al MO del ANDSF con su interfaz S14. En la Figura 9. puede verse el proceso de descubrimiento, consulta del ANQP encapsulado en el GAS. El dispositivo busca redes cercanas y descubre mas de una con la capacidad de manejar el HS2.0, envía un pedido información del ANQP que se encapsula en el GAS a cada una de ellas. Cada servidor responderá enviando una trama con la información requerida.

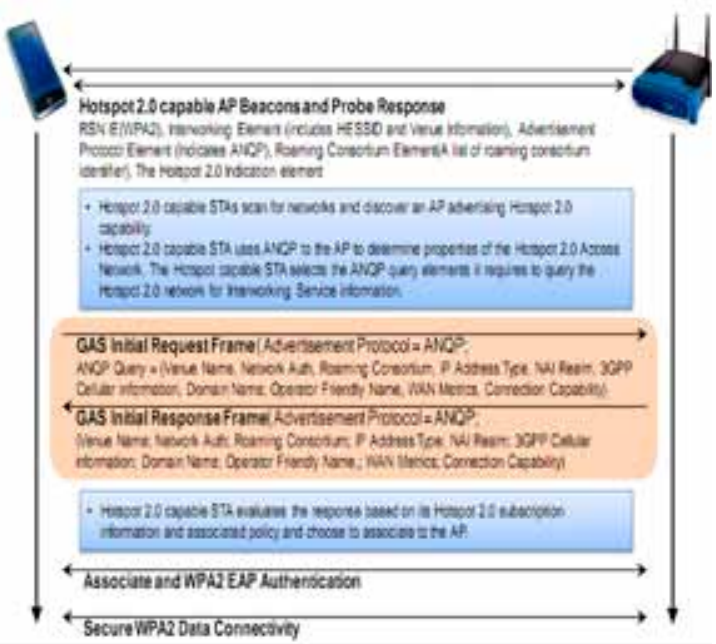

Figura 9. Proceso de conexión de HS2.0 con GAS-ANQP ${ }^{6}$

Con la información recolectada el HS2.0 del UE analiza los datos y se decide por la mejor conexión de acuerdo a las políticas que posee.

Este proceso, además de totalmente automático y con la posibilidad de utilizar los parámetros de autenticación contenidos en la SIM del UE, decide en base a un análisis de todos los datos del ANQP, que entre otros contienen, información de la red 3GPP si la hay, capacidades de confecciona y métricas de la WAN.

Estos dos últimos conjuntos de parámetros, dan la posibilidad al HS2.0 de selec- 
cionar la real mejor conexión, hasta llegar a la nube, asegurando una óptima velocidad de datos. La mejora comparada a los métodos de conexiona anteriores a HS2.0 es notable fundamentalmente en términos de QoE para el usuario y no menos importante en un uso racional de los recursos del operador.

\section{7 $\mathrm{La}$ arquitectura combinada $\mathrm{Wi}-\mathrm{Fi}$ celular [3][7]}

Las posibilidades de ANDSF en las redes celulares y el HS2.0 en las redes Wi$\mathrm{Fi}$, se combinan en una arquitectura que las contiene a partir del Release 12 (publicación 12) de 3GPP. Basada en ANDSF, la propuesta tiene cuatro componentes claves:

a - AP con HS2.0 habilitado a enviar a los UE la información necesaria para una óptima conexión por $\mathrm{Wi}-\mathrm{Fi}$.

b - Posibilidad de carga de tráfico desde distintos puntos de la red 3GPP dando en tiempo real la mejor opción.

c - El servidor ANDSF suministra las políticas al UE, incluyendo además de los parámetros descriptos en 2.4.1., la información de métricas de HS2.0 y cargas de la red 3GPP,

d - El UE utilizará esta información para seleccionar la mejor conexión o incluso si tiene posibilidad de conexión de 3GPP y WiFi simultáneas, seleccionar, en tiempo real, los caminos de los flujos de datos por donde convenga.

Para una mejor visión de esta arquitec- tura véase la Figura 10. donde puede entenderse la combinación de las tecnologías y la interconexión a la red de acceso 3GPP y WiFi a través del TWAG (Trusted Wireless Acces Gateway: Puerta de Acceso de Conexiones Inalámbricas Autenticadas). Se ven las posibilidades para dos tipos de UE, el de arriba es un dispositivo sin autentican por SIM (tabletas, netbooks) y el de abajo, identificado como ANDSF cliente es un típico teléfono inteligente (smartphone) con autenticación mediante SIM. Si la conexión es mediante el nodo $\mathrm{WiFi}$ y es autenticada, el tráfico solicitado por éste UE se encaminará por el TWAG accediendo al núcleo de la red del operador y aprovechando las conexiones de alta capacidad a la nube que éste posee. Un dispositivo como un smartphone, puede enviar tráfico por cualquiera de los puntos de accesos WiFi o Celular, o ambos a la vez.

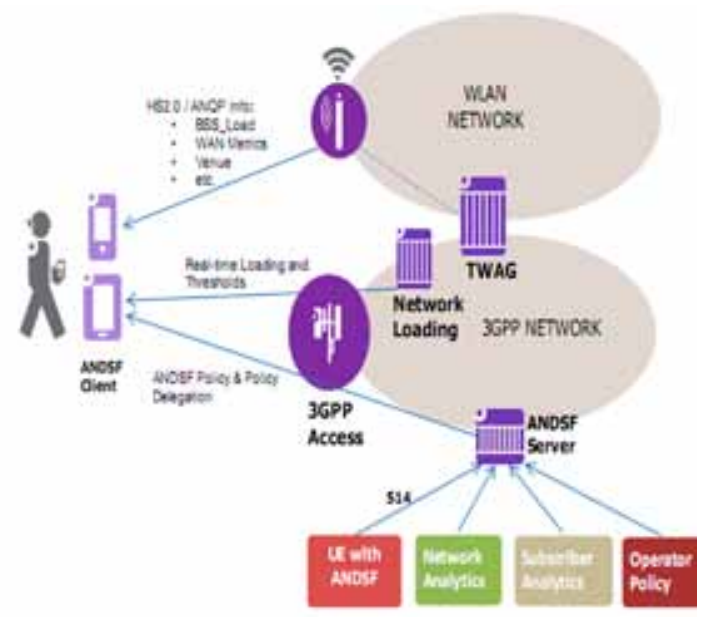

Figura 10. Envío dinámico de políticas ANDSF y control de acceso en tiempo real ${ }^{3}$

Nuevamente, el tráfico que éste curse por el acceso WiFi se encaminará a través del TWAG hacia la nube.

El hecho de tener un servidor como el 
ANDSF se piensa podría complicar el encaminamiento dentro del núcleo de la red del operador, es decir producir congestión. De todas maneras existen soluciones que no sólo evitarán esas posibles congestiones sino que aceleran y eficientizan el tráfico en el núcleo, a la vez de mejorar las bondades de ANDSF. Tal sistema se ve esquematizado en la Figura 11. Es lo que se conoce como una red SDN (Software Defined Network: Red Definida por Software), donde el protocolo que lleva adelante este sistema es el OpenFlow, desarrollado por la Open Net-

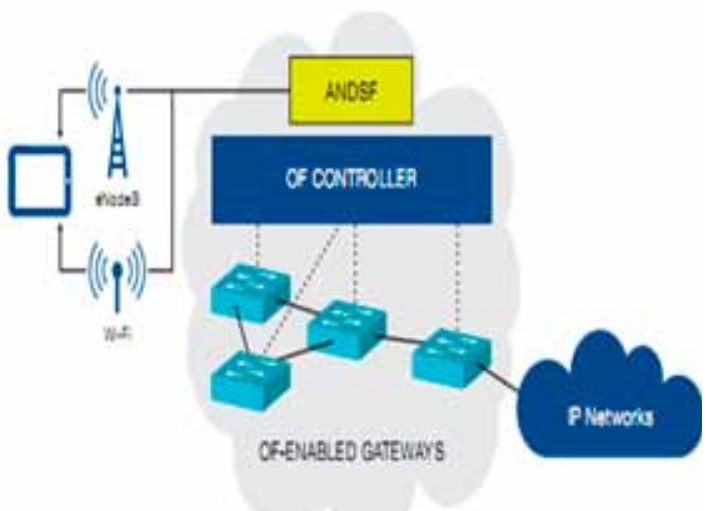

Figura 11. Tráfico del núcleo de la red móvil gestionado por OpenFlow ${ }^{7}$

working Foundation, que involucra a gran cantidad de compañías líderes en todo el espectro de la actividad de las comunicaciones de datos, por ejemplo IBM, Cisco, HP, Huawuei, ZTE, Nokia, etc. El objetivo de esta fundación es promocionar e incentivar el uso de las redes definidas por software a través del desarrollo de normas y estándares abiertos. La idea básica de SDN es separar las funciones de control y encaminamiento, dejando la tarea de encaminamiento a los dispositivos que ya lo hacen y el control, manejado por los mismos, hasta ahora a través de protocolos de enrutamiento, pasa a centralizarse en un servidor, que a través del protocolo OpenFlow $(O F)$, conoce el estado de la red en tiempo real, y envía la información de control a los switches para que encaminen el tráfico según las políticas de la SDN.

Así las aplicaciones avisan el servicio de flujo de datos requerido al servidor y éste, aplicando el conocimiento de la red actual y sus políticas definidas por el operador, envía las actualizaciones necesarias a los SW para que encaminen cada flujo de datos requerido según sus indicaciones. El SW conmuta por hardware a gran velocidad sólo interpretando las órdenes que figuran en las tablas actualizadas por OF. En el caso específico de las redes móviles como en la figura 11 el controlador de OF interactúa con el servidor ANDSF para re encaminar el tráfico de un móvil que pertenece a una conexión que se está congestionando. De la interacción deciden el camino y mientras OF reconfigura los SW, ANDSF busca el punto de acceso óptimo más próximo al UE para guiarlo a este en su traspaso de nodo (handover) sin cortar su sesión en ejecución, lo que se conoce como seamless-session (sesión sin cortes).

\section{CONCLUSIONES}

La integración de las redes Wi-Fi y celulares no es ya una opción como lo fue en un principio. En aquellos primeros pasos, el tráfico prioritario de la red celular como GSM y similares, era el tráfico de voz, que además en esa tecnología e incluso en $3 \mathrm{G}$ siguió siendo manejado como conmutación de circuitos. Por otra parte la comunicación de datos, incipiente en 2.5G(GSMGPRS), evolucionó rápidamente con $3 \mathrm{G}$ 
(UMTS) y las sucesivas mejoras de la interfaz de radio HSPA y posteriores. Esto junto a la posibilidad del acceso $\mathrm{WiFi}$ alternativo, a la evolución de los UE a equipos inteligentes (smartphones) dispararon la demanda de aplicaciones de datos de gran capacidad, sobre todo lo referente a streaming de audio y video. Con la llegada de 4G-LTE, ya la conmutación de circuitos desaparece, y todo el tráfico de voz, audio y video se resuelve como tráfico de datos (conmutación de paquetes). $4 \mathrm{G}$ tiene una mejora sustancial no solo en la interface de radio con OFDM, sino también en el manejo de tráfico IP exclusivamente. Se espera siga creciendo fuertemente la demanda de tráfico, pero el inconveniente de la red celular es el cubrimiento, no puede llegar con todo el potencial a todos lados. Las macro celdas, como se conocen a las celdas habituales tienen un alcance amplio pero decae su prestación en Mbps (Megabits por segundo) al irse hacia los bordes de su área de cobertura. Muchos casos de este empobrecimiento pueden cubrirse con celdas pequeñas (small cell) y la posibilidad de $4 \mathrm{G}$ de nodos de retransmisión (rely). Pero esto soluciona parte del problema. En áreas de mucha concentración y lugares cerrados amplios y concurridos, el servicio decae abruptamente. WiFi en cambio es fuerte en estos lugares, más aún con los nuevos estándares de mayor prestaciones como el 802.11 ac, y la implementación como se vió en párrafos anteriores del sistema de roaming de $\mathrm{WiFi}$, el HS2.0. Todo indica que la integración WiFi-Celular crecerá fuertemente para poder brindar en esta etapa una QoE muy elevada a los usuarios. La decisión de trabajar en conjunto con el fin de lograr una mejor integración de parte de los proveedores de los principales actores del mercado, ya ha logrado avanzar en el sentido de la integración con ANDSF y HS2.0. Pero ésta integración es desde el punto de vista del UE ya que estos servidores no intercambian información entre sí, y ese justamente es uno de los desafíos a resolver. En cuanto a la implementación de los operadores para aprovechar los avances logrados, dependerá del grado de inversión que decidan, de proveer nodos propios de WiFi con HS2.0 integrados a su núcleo, o integrar redes de este tipo de otros operadores para un funcionamiento conjunto. De darse una adecuada planificación en el sentido de aprovechar ambas redes, asistiremos en el futuro inmediato a una experiencia móvil de datos muy superior a la actual.

\section{LÍNEAS FUTURAS DETRABAJO}

Como ya se anticipo en párrafos anteriores, todavía queda mucho por hacer en cuanto a la integración WiFi-Celular. Los estándares de cada uno han avanzado mucho en pos de darle al UE la posibilidad de realizar una selección de red inteligente (INS: Intelligent Network Selection). E1 ANDSF utiliza información de las redes incluso $\mathrm{WiFi}$ para aportar parámetros de selección al UE. Pero esto no quiere decir que esten integrados los estándares. Esa, la comunicación de los servidores ANDSF y HS2.0 para intercambiar información de sus redes, es la línea de trabajo futura o inmediata para lograr una integración real y obtener una QoE mucho mayor y más estable y predecible. Mientras será necesario lograr que los fabricantes de equipos móviles incluyan el manejo de ANDSF en sus diseños y HLOS, como así también las aplicaciones necesarias para lograr una comunicación eficiente entre el HLOS y el $\mathrm{CM}$, optimizando la utilización de recursos y reduciendo las latencias en todo el proceso. 
5. Referencias

[1] Alcatel-Lucent: la explosión de datos entre 2011 y 2015 - Alberto Ríos, CTO de Alcatel-Lucent México - 2014

[2] $\mathrm{Wi}-\mathrm{Fi}$ and cellular integration: From $\mathrm{Wi}-$ Fi offload to

HetNets, M. Paolini, Senza Fili Wi-Fi and cellular integration - (C) 2014 Senza Fili Consulting, www.senzafiliconsulting.com .

[3] Integration of Cellular and $\mathrm{Wi}-\mathrm{Fi}$ networks, White paper, 4G AMERICAS (C) 2013 www.4gamericas.org .

[4] Wi-Fi roaming - building on ANDSF and Hotspot 2.0 - White paper, Alcatel-Lucent \& BT (C) 2012 . [5]http://www.etsi.org/deliver/etsi_ts/124300_ 124399/124312/12.07.00_60/

[6] IEEE 802 Enhanced nework detecion and selection, Max Riegel, IEEE 802.1 OmniRan TaskGroup

https://mentor.ieee.org/omniran/dcn/13/ omniran-13-0063-00-0000-ieee-802-gapsnetwork-detection-selection.pptx

[7] OpenFlow Enabled Mobile and Wireless Networks - Open Networking Foundation, 2013, https://www.opennetworking.org/ solution-brief-openflow-enabled-mobileand-wireless-networks 